\title{
Genome-wide genomic and functional association study for workability and calving traits in Holstein cattle
}

\author{
Michalina Jakimowicz ${ }^{1}$, Barbara Kosińska-Selbi ${ }^{1}$, Tomasz Suchocki 1, 2,* , Andrzej Zarnecki ${ }^{2}$, Wojciech Jagusiak ${ }^{2,3}$, \\ Małgorzata Morek-Kopeć 2,3, and Joanna Szyda 1,2 \\ 1 Biostatistics Group, Department of Genetics, Wrocław University of Environmental and Life Sciences, \\ Kożuchowska 7, 51-631 Wrocław, Poland; \\ 2 National Research Institute of Animal Production, Krakowska 1, 32-083 Balice, Poland; \\ 3 University of Agriculture in Krakow, Faculty of Animal Science, al. Mickiewicza 24/28, 30-059, Kraków, \\ Poland; \\ * Correspondence: tomasz.suchocki@upwr.edu.pl; Tel.: +48-71-3205758
}

\begin{abstract}
Simple Summary: Traits related to the calving abilities and workability of cows are essential both for the dairy economy and for the welfare of cows. We analyzed direct and maternal components of calving ease, direct and maternal components of stillbirth, milking speed, and temperament., by looking for SNPs, gene ontologies, and metabolic pathways significantly associated with additive genetic variability of those traits. On the genetic level, 150 significant SNPs were identified, while on the functional level we found 45 significant gene ontology terms and 5 metabolic pathways.
\end{abstract}

\begin{abstract}
Background: The goal of our study was to identify SNPs, metabolic pathways (KEGG), and gene ontology (GO) terms significantly associated with calving and workability; (2) Methods: Based on the EuroGenomics reference data set, we analyzed direct (DCE) and maternal (MCE) calving ease, direct (DSB), and maternal (MSB) stillbirth, milking speed (MSP), and temperament (TEM). We estimated SNP effects using a multi-SNP mixed-model. Further, SNP positions were mapped to genes, and GO terms/KEGG pathways of the corresponding genes were assigned. The estimation of GO term/KEGG pathway effects was based on a mixed-model using SNP effects as dependent variables; (3) Results: The number of significant SNPs comprised 59 for DCE, 25 for DSB and MSP, 17 for MCE and MSB, and 7 for TEM. Significant KEGG pathways were found for MSB (2), TEM (2), and MSP (1), while 11 GO terms were significant for MSP, 10 for DCE, 8 for DSB and TEM, 5 for MCE, and 3 for MSB.; (4) Conclusions: From the perspective of a better understanding of the genomic background of the phenotypes, traits with low heritabilities suggest that the focus should be moved from single genes to metabolic pathways or gene ontologies significant for the phenotype.
\end{abstract}

Keywords: GO: terms, GWAS, KEGG, SNP

\section{Introduction}

Traits related to the calving abilities and workability of cows are essential both for dairy economics and for the welfare of cows. According to Philipsson et al. [1] in 1994 only five countries - the Nordic countries and Slovenia, considered calving traits in their national selection indices. Nowadays already 20 countries carry out routine genetic evaluations of calving ease and 18 for workability (www.interbull.org). Despite generally low heritabilities, during the last decade, it has been possible to conduct successful selection, resulting in an improvement in calving ease and decrease of stillbirth rate, as demonstrated by Ma et al. [2]. It is thus interesting to explore the genetic architecture of these traits, by identifying polymorphisms associated with calving traits and even possibly to pinpoint the candidate genes. Numerous Genome-wide Association Studies (GWAS) for calving performance have been conducted so far, recently reviewed by Ma et al. [2]. On 
the other hand, traits related to handling cows on farms - summarised under the general term workability, have only recently been subjected to genetic evaluation, which is currently carried out by 18 countries on the international level (www.interbull.org). So far, only four GWAS results for workability traits have been published by Jardim et al. [3], Marete et al. [4], and Chen et al. [5] indicating that further studies on various populations are needed for a better understanding of the genetic components of workability phenotypes.

Therefore, the primary goal of our study was to identify SNPs significantly associated with the direct and maternal components of calving ease, direct and maternal components of stillbirth, cow temperament and milking speed. However, having in mind the low heritabilities of all those traits and then consequently lack of genes with very strong individual effects, we defined a secondary goal of the study which was the identification of metabolic pathways and gene ontology terms significantly associated with workability and calving. 


\section{Materials and Methods}

The analyzed data set originated from the EuroGenomics Holstein-Friesian dairy cattle population. For each bull, two workability and four calving traits, in the form of deregressed breeding values (DRP) corresponding to the Interbull evaluation from April 2020 were available. In particular, the analyzed traits comprised: direct calving ease (DCE), maternal calving ease (MCE), direct stillbirth (DSB), maternal stillbirth (MSB), milking speed (MSP), and temperament (TEM). The number of bulls used in the analysis together with heritabilities for each of the traits were summarized in Table 1.

Table 1 Number of bulls and heritability.

\begin{tabular}{lll}
\hline Trait $^{1}$ & Number of bulls & Heritability \\
\hline DCE & 30,603 & 0.05 \\
MCE & 29,738 & 0.04 \\
DSB & 24,521 & 0.03 \\
MSB & 28,081 & 0.05 \\
TEM & 22,301 & 0.09 \\
MSP & 28,376 & 0.12 \\
\hline
\end{tabular}

${ }^{1}$ DCE - Direct calving ease, MCE - Maternal calving ease, DSB - Direct stillbirth,

MSB - Maternal stillbirth, TEM - temperament, MSP - Milking speed

The majority of individuals (87\%) was genotyped by the Illumina BovineSNP50 BeadChip Version 2 containing 54,609 SNPs. Bulls genotyped with other platforms were imputed to the above using the Beagle software [6]. SNP preselection criteria comprised a minor allele frequency of at least 0.01 and technical quality of genotyping expressed by a minimum call rate of $99 \%$. After editing, 46,216 SNPs remained for further analysis.

The following mixed model was used to estimate the additive effects of SNPs: $\mathbf{y}=$ $\mathbf{X b}+\mathbf{Z}_{1} \mathbf{g}+\mathbf{Z}_{\mathbf{2}} \mathbf{a}+\mathbf{e}(\mathbf{1})$, where $\boldsymbol{y}$ represents a vector of DRPs for considered traits; $\boldsymbol{b}$ is a vector of fixed effects representing a general mean with a design matrix $\boldsymbol{X} ; \boldsymbol{Z}_{\mathbf{1}}$ is a design matrix for SNP genotypes, which is parameterized as $-1,0$, or 1 for a homozygous, heterozygous and an alternative homozygous SNP genotype, respectively; $\boldsymbol{g}$ is a vector of random additive SNP effects; $\boldsymbol{Z}_{2}$ is a design matrix for a residual polygenic effect; $\boldsymbol{a}$ is a vector of random bulls' residual additive polygenic effects, representing the part of additive polygenic variance which is not explained by SNPs; and $\boldsymbol{e}$ is a vector of residuals with $\boldsymbol{e} \sim \boldsymbol{N}\left(\mathbf{0}, \boldsymbol{D} \widehat{\boldsymbol{\sigma}}_{\boldsymbol{e}}^{2}\right)$, where $\boldsymbol{D}$ is a diagonal matrix containing the reciprocal of effective daughter contribution per bull on the diagonal and $\widehat{\sigma}_{e}^{2}$ representing the residual variance. The covariance structure of $\boldsymbol{g}$ was assumed to be $\boldsymbol{g} \sim \boldsymbol{N}\left(\mathbf{0}, \boldsymbol{I} \frac{\widehat{\sigma}_{\boldsymbol{a}}^{2}}{\boldsymbol{N}_{\boldsymbol{s} \boldsymbol{}}}\right)$, with $\boldsymbol{I}$ being an identity matrix and $\widehat{\boldsymbol{\sigma}}_{\boldsymbol{a}}^{2}$ representing the additive genetic variance of a given trait and NSNP is the number of SNPs. $\boldsymbol{a} \sim \boldsymbol{N}\left(\mathbf{0}, \boldsymbol{A} \widehat{\boldsymbol{\sigma}}_{\boldsymbol{a} *}^{2}\right)$, where $\boldsymbol{A}$ is the numerator relationship matrix and $\widehat{\boldsymbol{\sigma}}_{\boldsymbol{a} *}^{2}$ is a predetermined ratio of genetic variance for each trait. In this study $\widehat{\boldsymbol{\sigma}}_{\boldsymbol{a} *}^{2}=\mathbf{0 . 4} \cdot \widehat{\boldsymbol{\sigma}}_{\boldsymbol{a}}^{2}$.

The estimation of parameters of the above model was based on solving the mixed model
equations
$\left[\begin{array}{cccc}X^{T} R^{-1} X & X^{T} R^{-1} Z_{1} & X^{T} R^{-1} Z_{2} \\ Z_{1}^{T} R^{-1} X & Z_{1}^{T} R^{-1} Z_{1}+G_{1}^{-1} & Z_{1}^{T} R^{-1} Z_{2} \\ Z_{2}^{T} R^{-1} X & Z_{2}^{T} R^{-1} Z_{1} & Z_{2}^{T} R^{-1} Z_{2}+G_{2}^{-1}\end{array}\right]^{-1}\left[\begin{array}{c}X^{T} R^{-1} y \\ Z_{1}^{T} R^{-1} y \\ Z_{2}^{T} R^{-1} y\end{array}\right]$, 
where $\boldsymbol{R}=\boldsymbol{D} \widehat{\boldsymbol{\sigma}}_{\boldsymbol{e}}^{2}, \boldsymbol{G}_{1}=\boldsymbol{I} \frac{\widehat{\sigma}_{a}^{2}}{\boldsymbol{N}_{\text {snp }}}$ and $\boldsymbol{G}_{2}=\boldsymbol{A} \widehat{\boldsymbol{\sigma}}_{\boldsymbol{a}^{*}}^{2}$. Consequently, the variance of $\boldsymbol{y}$ is then given by $Z_{1} G_{1} \mathbf{Z}_{1}^{\mathrm{T}}+Z_{2} G_{2} \mathbf{Z}_{2}^{\mathrm{T}}+R$.

For testing the hypotheses $\left(\boldsymbol{H}_{\mathbf{0}}: \boldsymbol{g}=\mathbf{0}\right.$ vs. $\left.\boldsymbol{H}_{\mathbf{1}}: \boldsymbol{g} \neq \mathbf{0}\right)$, we used the Wald test: $\boldsymbol{W}=$ $\frac{\widehat{g}}{\boldsymbol{\sigma}_{\widehat{g}}}$, where $\boldsymbol{\sigma}_{\widehat{g}}$ is a standard error of the estimated SNP effect $\widehat{\boldsymbol{g}}$. Under $H_{0}$ this statistic follows the standard normal distribution. The multiple testing correction was carried out via the Bonferroni approach [8].

To associate SNPs with closest genes, all polymorphism coordinates from the Illumina BovineSNP50 BeadChip were remapped to the ARS_UCD1.2 Bos taurus reference genome using biomaRt [9] implemented in Bioconductor and the NCBI Remap tool. Then, genomic annotation of SNPs was done by using the Variant Effect Predictor software [10], with the maximum distance between an SNP and its closest gene set to 5,000 bp. The functional information was expressed by pathways defined by the Kyoto Encyclopedia of Genes and Genomes (KEEG) and by Gene Ontology (GO) terms corresponding to the genes marked by SNPs from the Illumina BovineSNP50 BeadChip. The DAVID software [11] was used for the annotation. The estimation of KEGG pathways and GO terms was carried out based on the following mixed model: $\boldsymbol{y}^{*}=\boldsymbol{u}+\boldsymbol{Z}^{*} \boldsymbol{p}+\boldsymbol{e}^{*}(\mathbf{2})$, where $\boldsymbol{y}^{*}$ is the absolute value of the SNP effect for a given trait; $\boldsymbol{p}$ is a random KEGG pathway or GO term effect and $\boldsymbol{e}^{*}$ represents the residual term. $\boldsymbol{Z}^{*}$ is the incidence matrix KEGG pathway or GO term effects $\boldsymbol{p}$. It is assumed that $\boldsymbol{p} \sim \boldsymbol{N}\left(\mathbf{0}, \boldsymbol{P} \widehat{\boldsymbol{\sigma}}_{\boldsymbol{p}}^{2}\right)$ and $\boldsymbol{e}^{*} \sim \boldsymbol{N}\left(\mathbf{0}, \boldsymbol{L} \widehat{\boldsymbol{\sigma}}_{\boldsymbol{e}^{*}}^{2}\right)$, where $\boldsymbol{P}$ is a covariance matrix between KEGG pathways or GO terms, consisting of the percentage of common genes between them and $\widehat{\sigma}_{p}^{2}$ represents KEGG pathway or GO term variance. $\boldsymbol{L}$ is a diagonal matrix of dimension NSNP $\times$ NSNP containing on the diagonal 1 (if an SNP was assigned a KEGG pathway or a GO term) or 10 (if an SNP was not assigned any functional element), $\widehat{\boldsymbol{\sigma}}_{\boldsymbol{e}^{*}}^{2}$ is the residual variance. For testing the hypotheses $\boldsymbol{H}_{\mathbf{0}}: \boldsymbol{p}=\mathbf{0}$ vs. $\boldsymbol{H}_{\mathbf{1}}: \boldsymbol{p} \neq \mathbf{0}$, the Wald test was used as defined above. Similar to Model (1), the Bonferroni multiple testing correction was used to modify the nominal P-values [8].

\section{Results}

\subsection{Direct calving ease}

For DCE the largest number of significant SNPs was detected among all analyzed traits. GWAS results, visualized in Figure 1, demonstrated an accumulation of 22 highly significant SNPs on chromosome 18, distributed across a region spanning 10,225,739 bp located close to the end of the chromosome. Significant SNPs were also identified on other chromosomes: BTA5, BTA7, BTA17, BTA19, BTA21, BTA25, and BTA29. The summary of significant SNP associations with DCE was presented in Supplemental Table S1. In this table, it is visible that the majority (except three within-gene doublets and 15 intergenic SNPs) of SNPs significant for direct calving ease marked a different gene, as a result, 41 genes can be considered as candidates. 


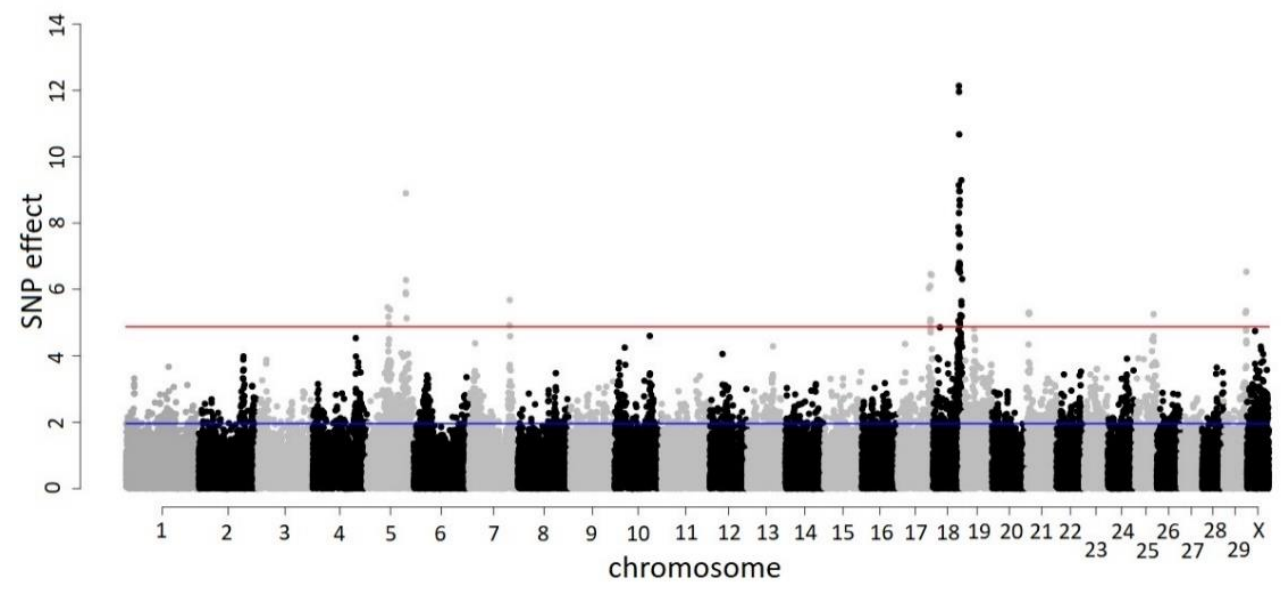

Figure 1 GWAS results for direct calving ease. The blue line represents a nominal, the red line a Bonferroni corrected critical value corresponding to the $5 \%$ significance level.

By moving the scope from genes to functions classified through GO terms, we identified seven significant biological processes: cartilage condensation, cell aggregation, artery development, nucleus localization, cardiac myofibril assembly, myofibril assembly, and actin filament-based movement. Microfilament motor activity was the significant molecular function, while two significant cell components were myosin complex and neuromuscular junction (Table 2). No metabolic pathway specific for the manifestation of direct calving ease phenotype was identified.

Table 2 Significant GO terms.

\begin{tabular}{llll}
\hline Trait & GO term & Effect & P-value \\
\hline \multirow{6}{*}{ DCE } & GO:0001502 & 4.8154 & $2.30 \cdot 10^{-3}$ \\
& GO:0098743 & 4.8154 & $2.30 \cdot 10^{-3}$ \\
& GO:0060840 & 8.6306 & $3.05 \cdot 10^{-18}$ \\
& GO:0000146 & 8.6306 & $3.05 \cdot 10^{-18}$ \\
& GO:0051647 & 8.6306 & $3.05 \cdot 10^{-18}$ \\
& GO:0055003 & 8.6306 & $3.05 \cdot 10^{-18}$ \\
& GO:0030239 & 8.6306 & $3.05 \cdot 10^{-18}$ \\
& GO:0016459 & 8.6306 & $3.05 \cdot 10^{-18}$ \\
& GO:0030048 & 8.6306 & $3.05 \cdot 10^{-18}$ \\
& GO:0031594 & 8.6306 & $3.05 \cdot 10^{-18}$ \\
& GO:1903319 & 4.1611 & $4.92 \cdot 10^{-2}$ \\
& GO:0099572 & 4.5622 & $7.90 \cdot 10^{-3}$ \\
MCE & GO:0050804 & 4.5622 & $7.90 \cdot 10^{-3}$ \\
& GO:0060076 & 4.5622 & $7.90 \cdot 10^{-3}$ \\
& GO:0014069 & 4.5622 & $7.90 \cdot 10^{-3}$ \\
& GO:0060840 & 4.6114 & $6.20 \cdot 10^{-3}$ \\
& GO:0000146 & 4.6114 & $6.20 \cdot 10^{-3}$ \\
& GO:0051647 & 4.6114 & $6.20 \cdot 10^{-3}$ \\
DSB & GO:0055003 & 4.6114 & $6.20 \cdot 10^{-3}$ \\
& GO:0030239 & 4.6114 & $6.20 \cdot 10^{-3}$ \\
& GO:0016459 & 4.6114 & $6.20 \cdot 10^{-3}$ \\
& GO:0030048 & 4.6114 & $6.20 \cdot 10^{-3}$ \\
\hline
\end{tabular}




\begin{tabular}{llll}
\hline \multirow{4}{*}{ MSB } & GO:0031594 & 4.6114 & $6.20 \cdot 10^{-3}$ \\
& GO:0005545 & 4.9310 & $1.30 \cdot 10^{-3}$ \\
& GO:0019933 & 5.2822 & $2.00 \cdot 10^{-4}$ \\
& GO:0019935 & 5.2822 & $2.00 \cdot 10^{-4}$ \\
& GO:0046717 & 5.5607 & $1.34 \cdot 10^{-8}$ \\
& GO:0015812 & 5.5607 & $1.34 \cdot 10^{-8}$ \\
& GO:0031901 & 5.5987 & $1.34 \cdot 10^{-8}$ \\
& GO:1902656 & 8.5417 & $6.61 \cdot 10^{-18}$ \\
& GO:0097553 & 8.5417 & $6.61 \cdot 10^{-18}$ \\
& GO:0044381 & 4.4921 & $1.10 \cdot 10^{-2}$ \\
& GO:2001275 & 4.4921 & $1.10 \cdot 10^{-2}$ \\
& GO:2001273 & 4.4921 & $1.10 \cdot 10^{-2}$ \\
& GO:0022834 & 6.8714 & $3.17 \cdot 10^{-12}$ \\
& GO:0015276 & 6.8714 & $3.17 \cdot 10^{-12}$ \\
& GO:0016788 & 4.4982 & $1.07 \cdot 10^{-2}$ \\
& GO:0008081 & 4.4982 & $1.07 \cdot 10^{-2}$ \\
& GO:0005509 & 4.4982 & $1.07 \cdot 10^{-2}$ \\
& GO:0035329 & 4.3080 & $2.56 \cdot 10^{-2}$ \\
& GO:0005545 & 4.6258 & $5.80 \cdot 10^{-3}$ \\
& GO:0005245 & 4.5538 & $8.20 \cdot 10^{-3}$ \\
& GO:0046514 & 5.0346 & $7.00 \cdot 10^{-4}$ \\
& GO:0046479 & 5.0346 & $7.00 \cdot 10^{-4}$ \\
& GO:0030149 & 5.0346 & $7.00 \cdot 10^{-4}$ \\
\hline
\end{tabular}

\subsection{Maternal calving ease}

For the maternal component of calving ease, much fewer significant SNPs (17) were identified than for the direct component (59). As is visible in Figure 2, the most of significant SNPs (8) were scattered over almost the entire length of BTX, from 863,652 bp up to $127,125,130 \mathrm{bp}$. All three SNPs from BTA18 were also significant for DCE, albeit with lower P-values.

Same as for DCE, no significantly associated metabolic pathways were identified, but five gene ontologies revealed a significant association with MCE, which were different than the ontologies significant for the direct component (Table 2). In particular, these were processes of positive regulation of protein maturation and modulation of chemical synaptic transmission with synapses being associated with cellular components. Also, for the maternal component of calving ease, no significant molecular pathway was determined. 


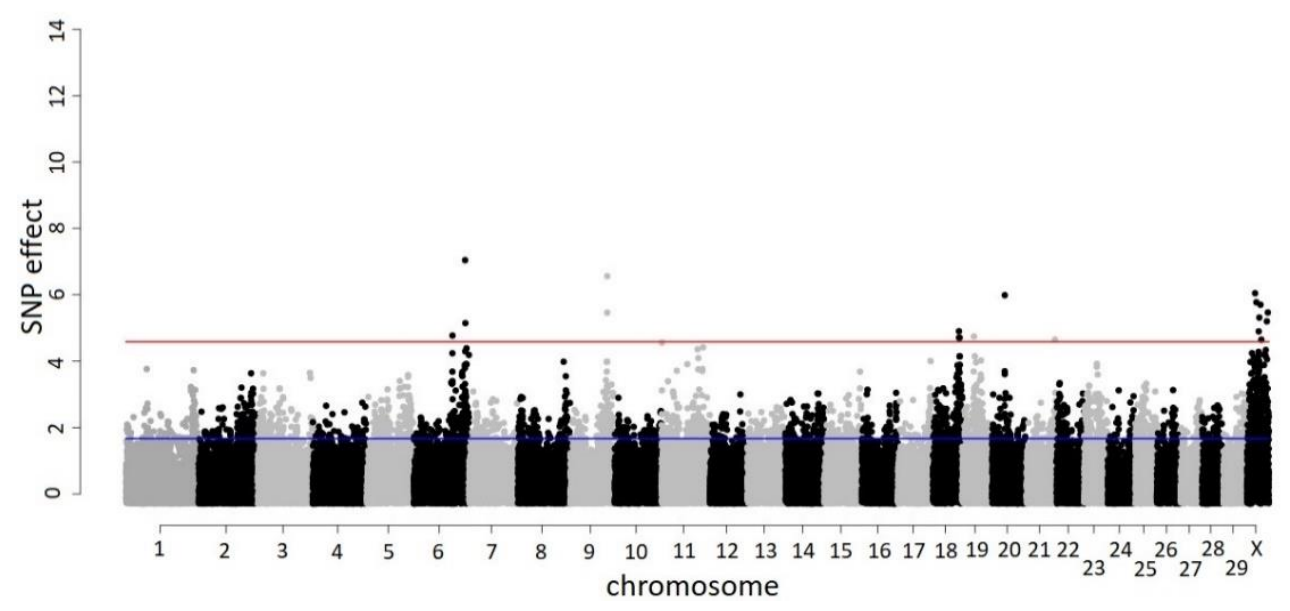

Figure 2 GWAS results for maternal calving ease. The blue line represents a nominal, the red line a Bonferroni corrected critical value corresponding to the $5 \%$ significance level.

\subsection{Direct stillbirth}

GWAS results for direct stillbirth were visualised in Figure 3. 19 out of the 25 significant SNPs overlapped with those that were also significant for DCE. Both traits were characterised by many significant SNPs located toward the end of BTA18 - except rs110508656, all the other 18 polymorphisms are pleiotropic for DSB and DCE. Another chromosome with multiple significant SNPs was the BTX, however, each of the four polymorphisms points out at a very different location spanning as many as $192,213,034$ bp. Moreover, single SNPs with a borderline significance were detected on BTA5 $(\mathrm{P}=0.0468)$ and BTA23 $(\mathrm{P}=0.0356)$. GWAS significant results and their annotations were summarised in Supplemental Table S1. The similarity of the genetic architecture underlying DSB and DCE revealed by GWAS was further reflected on the functional annotation level, since all eight GO terms significantly associated with direct stillbirth were also significant for DCE (Table 2). No significant association of stillbirth with molecular pathways was determined.

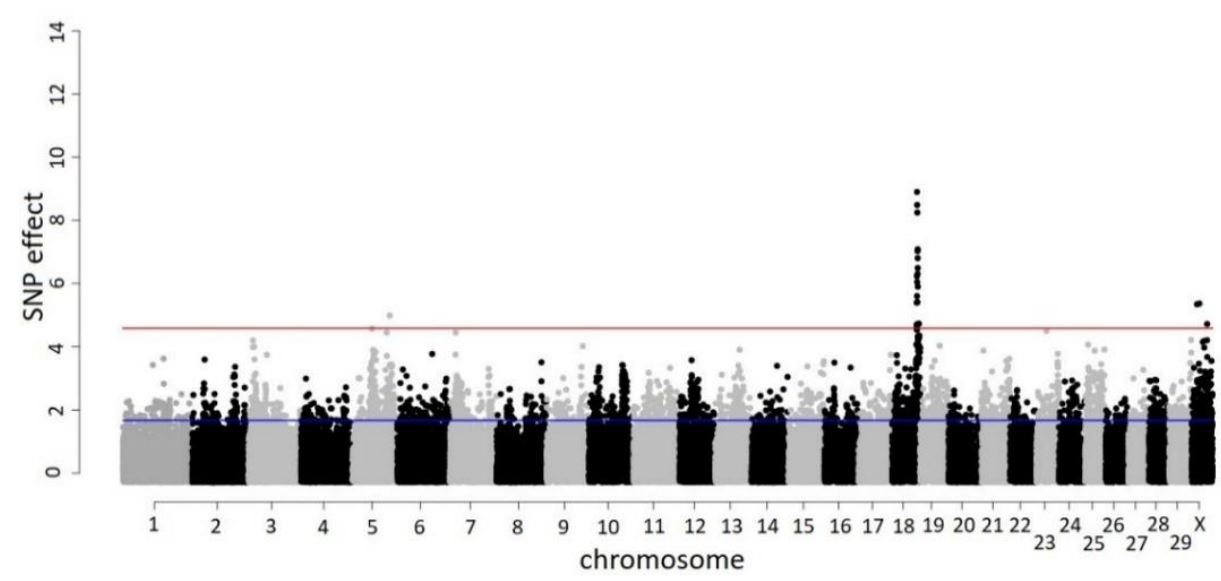

Figure 3 GWAS results for direct stillbirth. The blue line represents a nominal, the red line a Bonferroni corrected critical value corresponding to the $5 \%$ significance level. 
The largest number (seven) of SNPs significant for MSB was located on BTA9. Except for rs43615598, they also exhibited the highest effects on MSB among all significant SNPs. The three SNPs located on BTA18 were also significant for the direct component of stillbirth (as well as for DCE), five SNPs located on BTA9, BTA18, and BTX were also associated with the maternal component of calving ease. The summary on SNPs significant for MSB is given in Supplemental Table S1 and GWAS based Manhattan plot was presented in Figure 4. Signalling mediated by cyclic nucleotides (in particular by cAMP) was the biological process associated with the maternal component of stillbirth, while the significant molecular function comprises binding of 1-phosphatidylinositol (Table 2). MSB was the only calving-related trait for which a significant KEGG metabolic pathway could be assigned (Table 3): PD-L1 expression and PD-1 checkpoint pathway in cancer (bta:05235) and Th1 and Th2 cell differentiation (bta:04658).

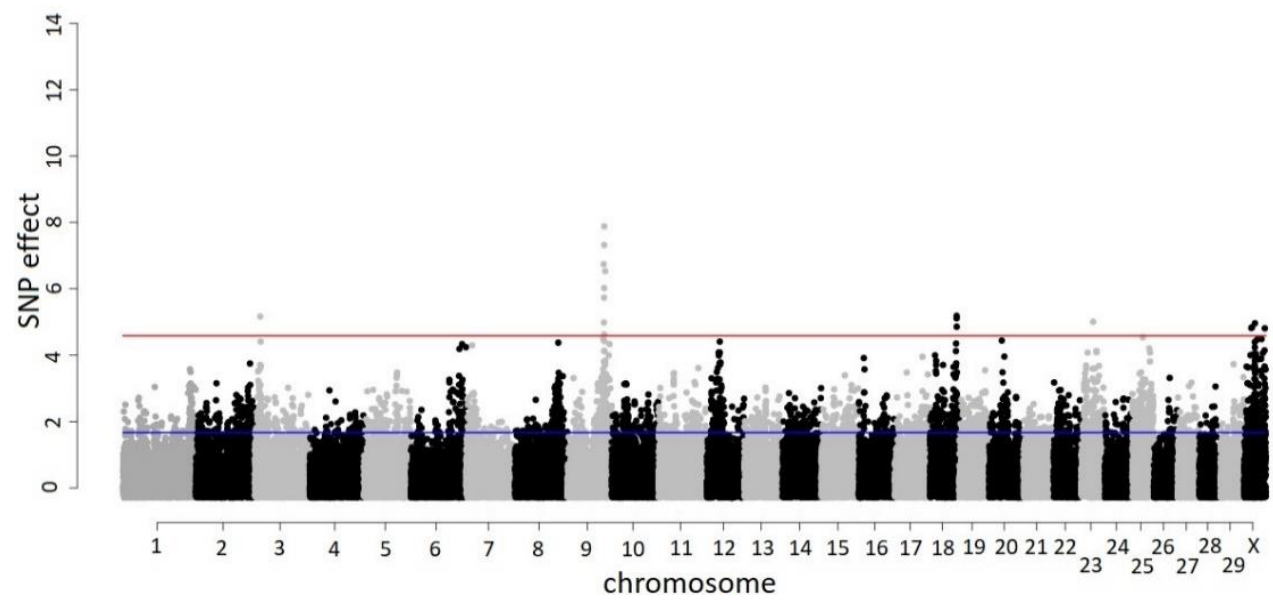

Figure 4 GWAS results for maternal stillbirth. The blue line represents a nominal, the red line a Bonferroni corrected critical value corresponding to the $5 \%$ significance level.

\subsection{Temperament}

All seven SNPs significant for temperament were located on chromosome $\mathrm{X}$ but across a very long span of 130,498,495 bp (Figure 5 and Supplemental Table S1). Eight gene ontology terms were estimated as significant for temperament, which are related to the processes of gamma-aminobutyric acid secretion and transport, the transmembrane import of calcium ions and glucose into cells as well as the early endosome membrane cell component (Table 2) The significant KEGG pathways related to the metabolism of porphyrin, chlorophyll, glyoxylate, and dicarboxylate (Table 3). 


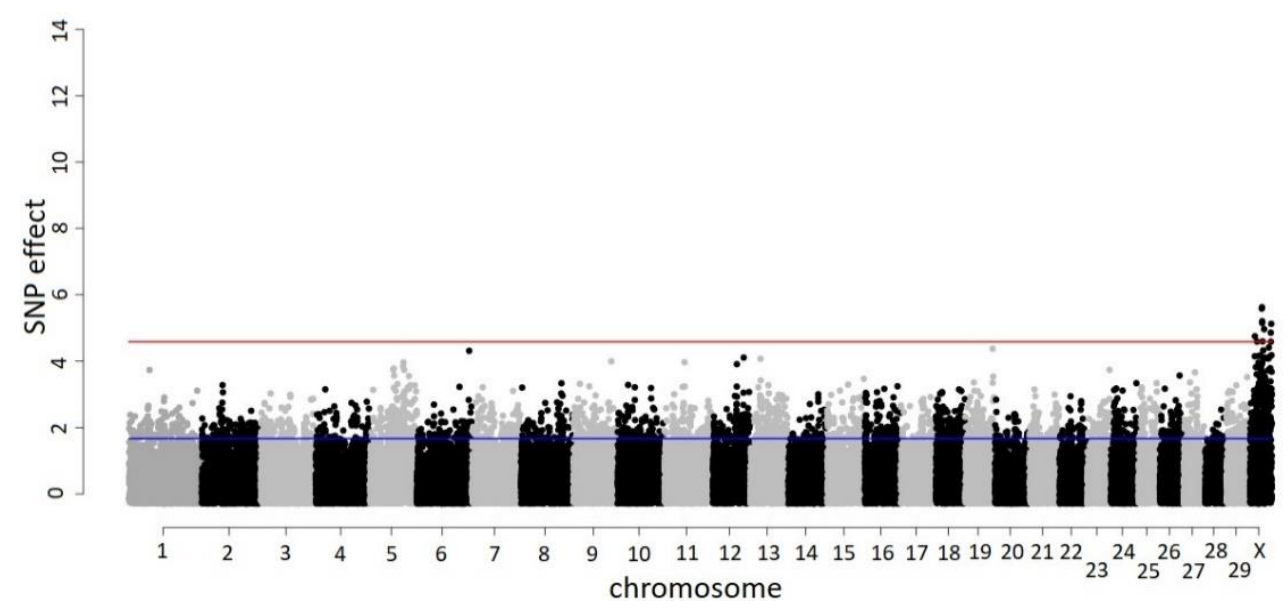

Figure 5 GWAS results for temperament. The blue line represents a nominal, the red line a Bonferroni corrected critical value corresponding to the $5 \%$ significance level.

\subsection{Milking speed}

With one exception, all 24 SNPs significantly associated with milking speed did not show pleiotropy with other traits considered in this study. The most SNPs (ten) were located on BTA19 with the most significant of them forming two groups. One, close to the beginning of the chromosome, between $7,250,802 \mathrm{bp}$ and $7,717,717 \mathrm{bp}$ harbouring two genes - ANKFN1 and a novel gene ENSBTAG00000038823. The other, close to the end of the chromosome, between 59,364,966 bp and 59,547,890 bp, harbouring a novel gene ENSBTAG00000048685. A group of five highly significant SNPs was also detected on BTA6, three of them within a short region, spanning 68,984 intergenic bps, close to the MSX1 gene. The summary and annotation of all significant SNPs were given in Supplemental Table S1, while the Manhattan plot of MSP was presented in Figure 6. Among the 11 gene ontology terms significantly influencing milking speed there are the biological processes related to hippo signalling as well as to the catalysis of ceramides and glycosphingolipids. The associated molecular functions comprised ligand-gated ion channel activity, phosphoric diester hydrolase activity, and voltage-gated calcium channel activity, as well as binding of calcium ions and 1-phosphatidylinositol (Table 2). The only significant KEGG pathway comprised the metabolism of degradation of valine, leucine and isoleucine (Table 3).

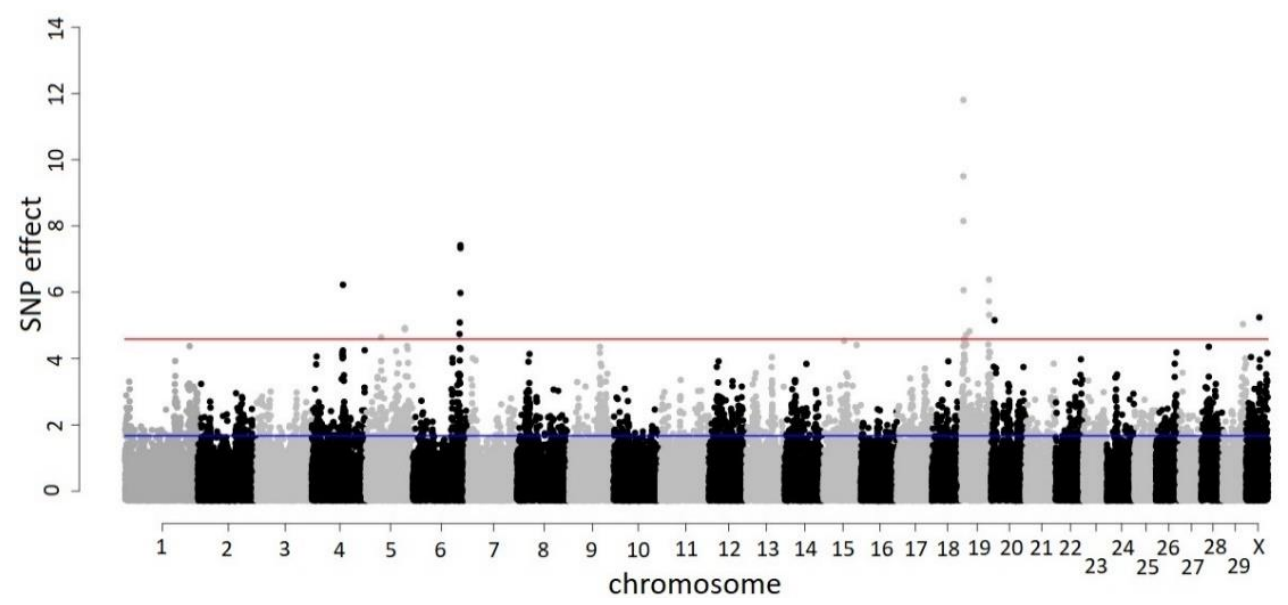

Figure 6 GWAS results for milking speed. The blue line represents a nominal, the red line a Bonferroni corrected critical value corresponding to the $5 \%$ significance level. 
Table 3 Significant KEGGs pathways.

\begin{tabular}{llrrrr}
\hline Trait & KEGG & Number of significant SNPs & Number of associated genes & Effect & P-value \\
\hline \multirow{2}{*}{ MSB } & bta:05235 & 62 & 29 & 3.6479 & $3.20 \cdot 10^{-2}$ \\
& bta:04658 & 62 & 29 & 3.6479 & $3.20 \cdot 10^{-2}$ \\
& bta:00630 & 34 & 15 & 3.7176 & $2.43 \cdot 10^{-2}$ \\
& bta:00860 & 21 & 13 & 3.7114 & $2.49 \cdot 10^{-2}$ \\
MSP & bta:00280 & 25 & 11 & 4.0403 & $6.50 \cdot 10^{-3}$ \\
\hline
\end{tabular}

\subsection{Pleiotropy}

The number of SNPs common across traits was visualised in Figure 7. No common SNPs with significant effects were identified between TEM and MSP, but among calving traits, some overlap, indicating a pleiotropic effect, was observed. A large number of 14 SNPs located on BTA18 were common between the direct components of calving ease and stillbirth. The maternal components of both traits share six SNPs located on BTA9, BTA18, and BTX.

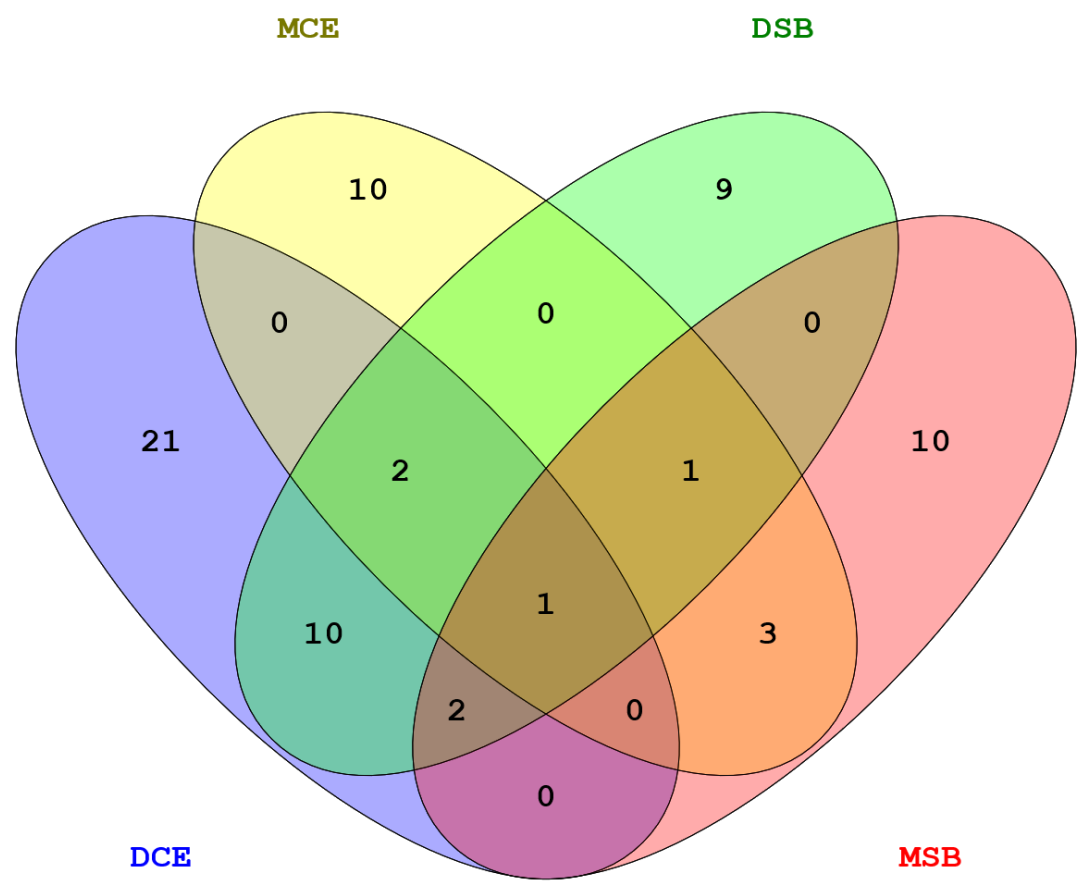

Figure 7 SNPS common among calving traits.

\section{Discussion}

Nine of the 22 significant SNPs detected for DCE on BTA18 were also reported as associated with direct calving ease by Müller et al. [12]: rs110389036, rs108984194, rs41582494, rs42843551, rs41664920, rs110889414, and rs110912084, as well as by Abo-Ismail et al. [13] - rs109478645 and rs109882115. For two other SNPs, significant associations with birth index and calf size, i.e. traits related to calving ease, were reported by Höglund 
et al. [14]. Furthermore, rs110312059 on chromosome 25 was previously reported as associated with direct calving ease by Sahana et al. [15]. Moreover, mouse orthologues of four genes marked by significant SNPs were associated with disease phenotypes that may be regarded as analogous to calving ease - embryonic growth retardation as well as embryonic, fetal and pre-weaning lethality.

Regarding MCE, among the significant SNPs on BTX, two have already been previously reported by Cole et al. [16] as associated with maternal calving ease - rs42766480 and direct calving ease - rs41567624. Among other significant SNPs an association with direct calving ease was reported for rs109399965 on BTA6, rs41601571 on BTA20 by Sahana et al. [16]. It is noteworthy that many of the significant SNPs were reported to be associated with moderately to highly heritable conformation traits, which may then indirectly impact calving ease in a maternal inheritance mode, i.e. body depth $\left(\mathrm{h}^{2}=0.21,2\right.$ SNPs), dairy form (1 SNP), rump width ( $\left.\mathrm{h}^{2}=0.30,5 \mathrm{SNPs}\right)$, stature ( $\left.\mathrm{h}^{2}=0.54,2 \mathrm{SNPs}\right)$, type (3 SNPs). Summary of significant SNPs was given in Supplemental Table S1.

Five SNPs from BTA18 significant for DSB were already reported for direct stillbirth by other studies: rs109478645 with the 2nd and rs109882115 with the 3rd overall highest effect by Höglund et al. [14], rs41664920, rs110889414, and rs110508656 by Müller et al. [12]. The last polymorphism is located within the tweety family member 1 gene (TTYH1) whose mouse orthologue is associated with embryonic lethality and embryonic growth arrest disorders. One of the four significant SNPs located on BTX - rs110344484, was also reported as associated with DSB by Cole et al. [16].

Differently from the previously described traits, in the case of GWAS results for MSB, no significant SNP was reported in the literature as associated with the trait. However, a significant SNP on BTA25 was located within the calcineurin-like EF-hand protein 2 gene (CHP2), which in the mouse is associated with pre-weaning lethality. Both KEGG pathways significant for MSB are related to the immune response, through T-type lymphocytes.

Regarding workability traits, the ATPase copper transporting alpha gene (ATP7A) harbouring rs41623769 which was one of the seven SNPs significant for TEM with the highest effect on temperament, is associated with temperament-related phenotypes of lethargy and hypoactivity in the mouse. One of the SNPs significant for MSP on BTA19 (rs29020026) was reported as associated with several udder conformation traits, such as front and rear teat placement, udder attachment, udder cleft, udder depth and height by Cole et al. [16] and rs41257416 from BTA5 was previously reported by Jardim et al. [3] as associated with milking speed.

Pleiotropic effects of SNPs observed in our study could have been expected based on high genetic correlations estimated for Norwegian Red cattle by Heringstad et al. [17] between direct components of stillbirth and calving difficulty, as well as between the maternal components of these traits, that amounted to 0.79 and 0.62 respectively.

\section{Conclusions}

Calving traits are low heritable. Their additive genetic component does not typically exceed 10\% (see our results in Table 1 and a recent review of Ma et al [2]. Therefore, the presence of single genes with marked effects is not to be anticipated for the traits. However, a surprisingly large overlap was observed between genomic regions, so many polymorphisms detected as significant in our study have already been indicated by previous analyses listed in the Results and Discussion section. This observation is important for carrying out the genomic selection on the traits, especially by incorporating targeted polymorphisms into the evaluation model. Still, from the basic perspective of a better understanding of the genomic background of the phenotypes, traits' low heritabilities indicate that the focus should be moved from single genes to metabolic pathways or gene ontologies significant for the manifestation of calving problems. Such a functionally based approach has not so far been considered in the relation to calving nor workability traits. 
Supplementary Materials: The following supporting information can be downloaded at: www.mdpi.com/xxx/s1, Supplementary table S1: SNP significant in GWAS and their annotation.

\begin{abstract}
Author Contributions: Conceptualization, Tomasz Suchocki and Joanna Szyda; Data curation, Andrzej Zarnecki; Formal analysis, Michalina Jakimowicz, Barbara Kosińska-Selbi and Tomasz Suchocki; Investigation, Andrzej Zarnecki, Wojciech Jagusiak and Małgorzata Morek-Kopeć; Methodology, Tomasz Suchocki and Joanna Szyda; Resources, Andrzej Zarnecki, Wojciech Jagusiak and Małgorzata Morek-Kopeć; Visualization, Michalina Jakimowicz and Barbara Kosińska-Selbi; Writing - original draft, Michalina Jakimowicz, Barbara Kosińska-Selbi and Tomasz Suchocki; Writing - review \& editing, Michalina Jakimowicz, Tomasz Suchocki and Joanna Szyda.
\end{abstract}

Funding: This research received no external funding.

Data Availability Statement: The datasets analysed for this study are available upon a formal request to the EuroGenomics cooperative.

Acknowledgments: Calculations have been carried out using resources provided by Wroclaw Centre for Networking and Supercomputing (http://wcss.pl), grant No. 509.

Conflicts of Interest: The authors declare no conflict of interest.

\title{
References:
}

1. Philipson, J.; Banos, G.; Arnason, T. Present and Future Uses of Selection Index Methodology in Dairy Cattle. J Dairy Sci 1994, 77, 3252-3261. https://doi.org/10.3168/jds.s0022-0302(94)77266-0.

2. Ma, L.; Cole, J.B.; Da, Y.; VanRaden, P.M. Symposium Review: Genetics, Genome-Wide Association Study, and Genetic Improvement of Dairy Fertility Traits. J Dairy Sci 2019, 102, 3735-3743, doi:10.3168/jds.2018-15269.

3. Jardim, J.G.; Guldbrandtsen, B.; Lund, M.S.; Sahana, G. Association Analysis for Udder Index and Milking Speed with Imputed Whole-Genome Sequence Variants in Nordic Holstein Cattle. J Dairy Sci 2018, 101, 2199-2212, doi:10.3168/jds.2017-12982.

4. Marete, A.; Sahana, G.; Fritz, S.; Lefebvre, R.; Barbat, A.; Lund, M.S.; Guldbrandtsen, B.; Boichard, D. Genome-Wide Association Study for Milking Speed in French Holstein Cows. J Dairy Sci 2018, 101, 6205-6219, doi:10.3168/jds.2017-14067.

5. Chen, S.-Y.; Oliveira, H.R.; Schenkel, F.S.; Pedrosa, V.B.; Melka, M.G.; Brito, L.F. Using Imputed Whole-Genome Sequence Variants to Uncover Candidate Mutations and Genes Affecting Milking Speed and Temperament in Holstein Cattle. J Dairy Sci 2020, 103, 10383-10398, doi:10.3168/jds.2020-18897.

6. Browning, B.L.; Browning, S.R. Genotype Imputation with Millions of Reference Samples. Am. J. Hum. Genet. 2016, 98, 116-126, doi:10.1016/j.ajhg.2015.11.020.

7. Henderson, C.R. Applications of Linear Models in Animal Breeding; Univ. of Guelph: Guelph, 1984; ISBN 9780889550308.

8. Dunnett, C.W. A Multiple Comparison Procedure for Comparing Several Treatments with a Control. J Am Stat Assoc 1955, 50, 1096-1121, doi:10.1080/01621459.1955.10501294.

9. Durinck, S.; Spellman, P.T.; Birney, E.; Huber, W. Mapping Identifiers for the Integration of Genomic Datasets with the R/Bioconductor Package BiomaRt. Nat Protoc 2009, 4, 1184-1191, doi:10.1038/nprot.2009.97.

10. McLaren, W.; Gil, L.; Hunt, S.E.; Riat, H.S.; Ritchie, G.R.S.; Thormann, A.; Flicek, P.; Cunningham, F. The Ensembl Variant Effect Predictor. Genome Biol 2016, 17, 122, doi:10.1186/s13059-016-0974-4.

11. Huang, D.W.; Sherman, B.T.; Lempicki, R.A. Bioinformatics Enrichment Tools: Paths toward the Comprehensive Functional Analysis of Large Gene Lists. Nucleic Acids Research 2009, 37, 1-13, doi:10.1093/nar/gkn923.

12. Müller, M.-P.; Rothammer, S.; Seichter, D.; Russ, I.; Hinrichs, D.; Tetens, J.; Thaller, G.; Medugorac, I. Genome-Wide Mapping of 10 Calving and Fertility Traits in Holstein Dairy Cattle with Special Regard to Chromosome 18. J Dairy Sci 2017, 100, 19872006, doi:10.3168/jds.2016-11506.

13. Abo-Ismail, M.K.; Brito, L.F.; Miller, S.P.; Sargolzaei, M.; Grossi, D.A.; Moore, S.S.; Plastow, G.; Stothard, P.; Nayeri, S.; Schenkel, F.S. Genome-Wide Association Studies and Genomic Prediction of Breeding Values for Calving Performance and Body Conformation Traits in Holstein Cattle. Genet Sel Evol 2017, 49, 82, doi:10.1186/s12711-017-0356-8.

14. Höglund, J.K.; Guldbrandtsen, B.; Lund, M.S.; Sahana, G. Analyzes of Genome-Wide Association Follow-up Study for Calving Traits in Dairy Cattle. BMC Genet 2012, 13, 71, doi:10.1186/1471-2156-13-71.

15. Sahana, G.; Guldbrandtsen, B.; Lund, M.S. Genome-Wide Association Study for Calving Traits in Danish and Swedish Holstein Cattle. J Dairy Sci 2011, 94, 479-486, doi:10.3168/jds.2010-3381.

16. Cole, J.B.; Wiggans, G.R.; Ma, L.; Sonstegard, T.S.; Lawlor, T.J.; Crooker, B.A.; Van Tassell, C.P.; Yang, J.; Wang, S.; Matukumalli, L.K.; et al. Genome-Wide Association Analysis of Thirty One Production, Health, Reproduction and Body Conformation Traits in Contemporary U.S. Holstein Cows. BMC Genomics 2011, 12, 408, doi:10.1186/1471-2164-12-408.

17. Heringstad, B.; Chang, Y.M.; Svendsen, M.; Gianola, D. Genetic Analysis of Calving Difficulty and Stillbirth in Norwegian Red Cows. J Dairy Sci 2007, 90, 3500-3507, doi:10.3168/jds.2006-792. 